\title{
Dynamic Compensation for Two-Axis Robot Wrist Force Sensors
}

\author{
Junqing Ma, Aiguo Song, and Dongcheng Pan \\ Jiangsu Key Lab of Remote Measurement and Control, School of Instrument Science and Engineering, Southeast University, \\ Nanjing 210096, China \\ Correspondence should be addressed to Aiguo Song; a.g.song@seu.edu.cn
}

Received 10 June 2013; Accepted 26 June 2013

Academic Editor: Guangming Song

Copyright ( 2013 Junqing Ma et al. This is an open access article distributed under the Creative Commons Attribution License, which permits unrestricted use, distribution, and reproduction in any medium, provided the original work is properly cited.

To improve the dynamic characteristic of two-axis force sensors, a dynamic compensation method is proposed. The two-axis force sensor system is assumed to be a first-order system. The operation frequency of the system is expanded by a digital filter with backward difference network. To filter high-frequency noises, a low-pass filter is added after the dynamic compensation network. To avoid overcompensation, parameters of the proposed dynamic compensation method are defined by trial and error. Step response methods are utilized in dynamic calibration experiments. Compared to experiment data without compensation, the response time of the dynamic compensated data is reduced by 30\% 40\%. Experiments results demonstrate the effectiveness of our method.

\section{Introduction}

Multiaxis robot wrist force sensors are necessary for robotic systems in which contact force information between robots and environments needs to be obtained. There are various kinds of multiaxis force sensors available in commercial and research area, for example, cross-beam type multiaxis force sensors [1,2], piezoelectric multiaxis force sensors [3], fiber multiaxis force sensors [4], and so on [5]. Multiaxis robot wrist force sensors are always mounted on the wrists of robots to convert multidimensional contact force signals into multichannel voltage signals. Such kinds of applications can be frequently found in assemble robots, teleoperation robotic systems, rehabilitation robots, and so forth [6-9].

During a robot task, the effectiveness of on-line force perception and feedback highly relies on the performances of the multiaxis robot wrist force sensor. The strong real time and rapidity in robot tasks require multiaxis force sensors to perform high dynamic characteristic. However, multiaxis force sensors (hereafter referred to as "force sensors") always have low natural frequency and small damping ratio owing to the low stiffness of elastic body and using of strain gauges. As a result, the dynamic response of the force sensors is more than $0.2 \mathrm{~ms}$, and the adjusting time is relatively long [10]. The A/D converters for force sensors will prolong the response time as well. The disparity of dynamic requirements from robotic tasks and the current performances of force sensors motivate the need to improve dynamic characteristics of force sensors. Improving dynamic characteristic of force sensors by hardware is limited and costly. In the field of measurement, dynamic performances of sensors are often improved by algorithms. Hence, dynamic compensation algorithms need to be designed to improve dynamic behavior of force sensors. Altintas and Park in [11] designed dynamic compensation algorithms by a Kalman filter for a spindle-integrated force sensor. $\mathrm{Xu}$ and $\mathrm{Li}$ in $[10,12]$ designed dynamic compensation algorithms by functional link artificial neural network (FLANN) for six-axis wrist force/torque sensor. Yu et al. in [13] designed dynamic compensation algorithms by genetic neutral network for robot wrist force sensor, but the artificial neural network is slow to converge and may be subjected to local minimum [14].

In this paper, we proposed a dynamic compensation method based on a digital filter with backward difference design. Because there are high-frequency noises in the output voltages of force sensors, a low-pass filter is added in the dynamic compensation system. Dynamic calibration experiments are conducted, in which step-response method is utilized. During the dynamic calibration experiments, a twoaxis force sensor which is designed and fabricated in our lab is used. Step signals of force are generated, while corresponding step responses of the two-axis force sensor are recorded by 


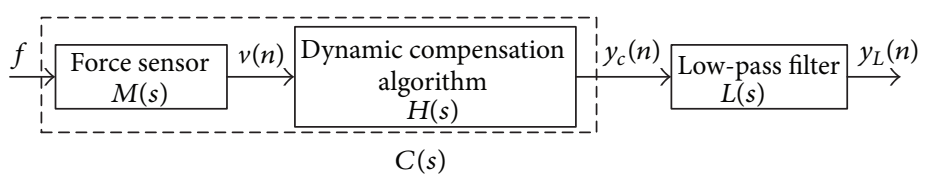

FIGURE 1: Flowchart of dynamic compensation of two-axis force sensors.

high-frequency data acquisition card. The proposed dynamic calibration method is applied to the dynamic calibration experiment data. The response time is greatly reduced with our method. The experiment results demonstrated the correctness and effectiveness of our method.

\section{Dynamic Compensation System}

2.1. Principle of Dynamic Compensation. The original operation frequency band of force sensors is too narrow to cover all the frequency components of input force signals. This causes the decays of high frequency components. Furthermore, dynamic behavior of force sensors will be decreased. In order to broaden the operation frequency, in this paper, a dynamic compensation part is added in force-sensor systems. With this method, dynamic performance of force sensors can be improved. Dynamic compensation of input forces in each dimension is done separately. Figure 1 shows the flowchart of the dynamic compensation of force sensors.

In Figure $1, f$ is the input force signal, and $v(n)$ is the output voltage of the two-axis force sensors. $y_{c}(n)$ is the results of $v(n)$ passing dynamic compensation network, and $y_{L}(n)$ is the dynamic compensated data after low-pass filter. $M(s)$ represents the transfer function of the force sensor, $H(s)$ represents the transfer function of the dynamic compensation, $L(s)$ represents the transfer function of the low-pass filter, and $C(s)$ represents the transfer function of the whole force sensor system.

We have

$$
C(s)=M(s) H(s) .
$$

2.2. Dynamic Compensation Algorithm. The force sensor system, as shown in Figure 1, is assumed as a first-order system. The transfer function of the force sensor, $M(s)$, can be expressed as

$$
M(s)=\frac{1}{1+\tau s} e^{-\lambda s},
$$

where $\tau$ is the time constant of the first-order system and $\lambda$ is the lag time of the force sensor.

The cut-off angular frequency of the force sensor $\omega$ can be calculated as

$$
\omega=\frac{1}{\tau} .
$$

If the frequency band of the force sensor is broadened to $k$ times, the cut-off angular frequency becomes $\omega_{c}=k \omega$. Then, $C(s)$ can be expressed as

$$
C(s)=\frac{1}{1+(\tau / k) s} e^{-\lambda s} .
$$

Combinations of (1), (2), and (4) lead to

$$
H(s)=\frac{C(s)}{M(s)}=\frac{1+\tau s}{1+(\tau / k) s} .
$$

After A/D converter, the output voltages signals of force sensors are digital signals. Equivalent digital filter in $z$ domain $H(z)$ can be obtained from (5) and backward difference method as shown in (6):

$$
s=\frac{1}{\tau_{\text {sam }}}\left(1-z^{-1}\right),
$$

where $\tau_{\text {sam }}$ is the sampling interval.

Combination of (5) and (6) leads to

$$
H(z)=k \frac{1+c \tau_{\mathrm{sam}}-z^{-1}}{1+b \tau_{\mathrm{sam}}-z^{-1}}=\frac{Y_{c}(z)}{X(z)},
$$

where $c=1 / \tau, b=k / \tau=k c$.

The difference equation of (7) can be calculated as

$$
\begin{aligned}
y_{c}(n)=\frac{1}{1+b \tau_{\text {sam }}}[ & k\left(1+c \tau_{\text {sam }}\right) x(n) \\
& \left.-k x(n-1)+y_{c}(n-1)\right] .
\end{aligned}
$$

Equation (8) is the dynamic compensation algorithm of force sensors. The proposed dynamic algorithm is simple and fast. It can be easily realized in software.

2.3. Digital Low-Pass Filter. As mentioned in the former section, the operation frequency of the force sensors is broadened in the proposed dynamic compensation algorithm. However, the extension of operation frequency will intensify high-frequency noises in the output voltage signals in each dimension. As a result, a low-pass filter is added after the dynamic compensation part, as shown in Figure 1, to filter out high frequency noise signals.

The common low-pass filter, called "moving average filter," is utilized. Moving average filter is able to reduce random noises while retaining a sharp step response. As a result, moving average filter is the premier filter for time domain encoded signals with random noises. The moving average filter operates by averaging a number of points from input signals to produce every point in output signals [15].

In the dynamic compensation process, a moving average signal average $m$ numbers of points in $y_{c}(n)$, as shown in (9):

$$
y_{L}(n)=\frac{1}{m_{i}} \sum_{i=n+1}^{n} y_{c}(n+i), \quad n=0,1,2 \ldots
$$




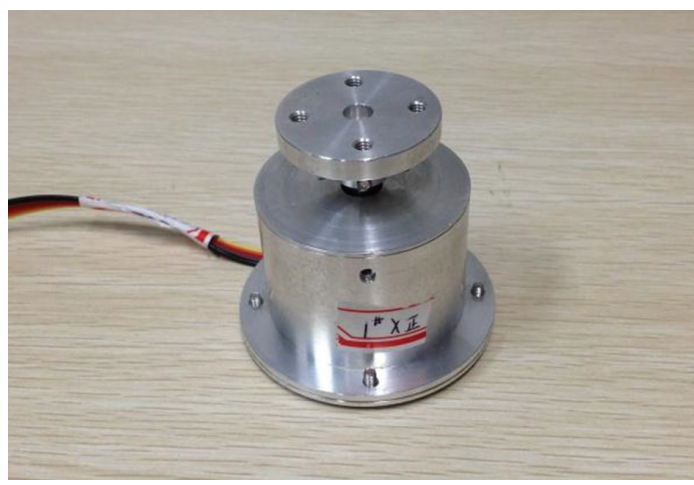

FIGURE 2: Prototype of a two-axis force sensor.

\section{Dynamic Compensation Results}

3.1. Dynamic Calibration Experiments. A prototype two-axis force sensor which is designed and fabricated in our lab is used in the dynamic calibration experiments, as shown in Figure 2.

The key component of the force sensor is the cross-beam elastic body. When external forces are applied, the crossbeam elastic body will be deformed. Eight strain gauges which are pasted on the cross-beam elastic body of the force sensor will detect the deformation and convert the variations of input forces into variations of resistances. Each four strain gauges are connected to establish a Wheatstone bridge circuit. Variations of resistances of strain gauges can be converted into variations of voltages. The voltages are amplified by amplifying circuit [16].

The two-axis force sensor is able to measure horizontal forces in both $\mathrm{X}$ direction and $\mathrm{Y}$ direction. Because of the symmetrical characteristic of the two-axis force sensor, we only do dynamic calibration experiment in $\mathrm{X}$ direction.

There are three kinds of common dynamic calibration methods for sensors, namely, the frequency method, the stepresponse method, and the impact response method [12]. As for force sensors, a sine wave input force signal is difficult to generate. An impact force can be generated by a hammer with a piezoelectric sensor $[17,18]$. However, the knocking position and angle are hard to control. Hence, step-response method [19] is preferred in dynamic calibration experiments for force sensors.

Figure 3 shows the platform of the dynamic calibration experiment setup.

As shown in Figure 3, a two-axis force sensor is mounted on an indexing plate in the center of the calibration table. The indexing plate can be rotated to ensure the directions of the loading forces. External horizontal forces are generated by pulley, nylon ropes and weights.

The cutting place of the nylon rope will be cut by a pair of scissors during the calibration experiment. When the nylon rope which is near the force sensor is cut, weights will fall down to the ground. A negative step force is generated. The cutting process should be quick and decisive.

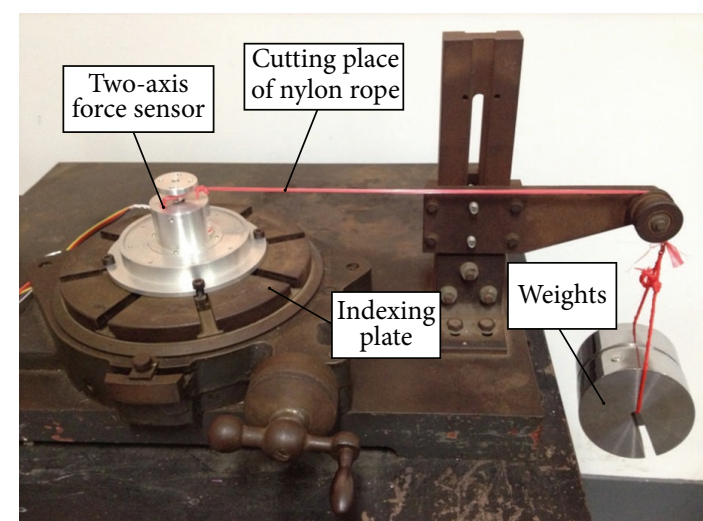

FIGURE 3: Dynamic compensation experiments setup.



FIGURE 4: Original step response data from dynamic compensation experiments.

The response of the two-axis force sensor is recorded by a data acquisition card with a sampling frequency of $250 \mathrm{KHz}$.

3.2. Results. The whole dynamic calibration experiment is repeated three times. The best experiment data which show the fewest vibrations are chosen. Figure 4 shows the original negative step response data recorded by the data acquisition card.

The calibration experiment data support the assumption that the two-axis force sensor is a first-order system. The transfer function of the force sensor can be expressed in (2). The time constant $\tau=1 \mathrm{~ms}$, and the sampling interval $\tau_{\text {sam }}=$ $0.004 \mathrm{~ms}$.

In the dynamic compensation network, parameters in the dynamic compensation algorithm and low-filter pass filter are defined by trial and error. Finally, the factor $k$ is defined as 3 ; that is, the operation frequency is expanded three times. 


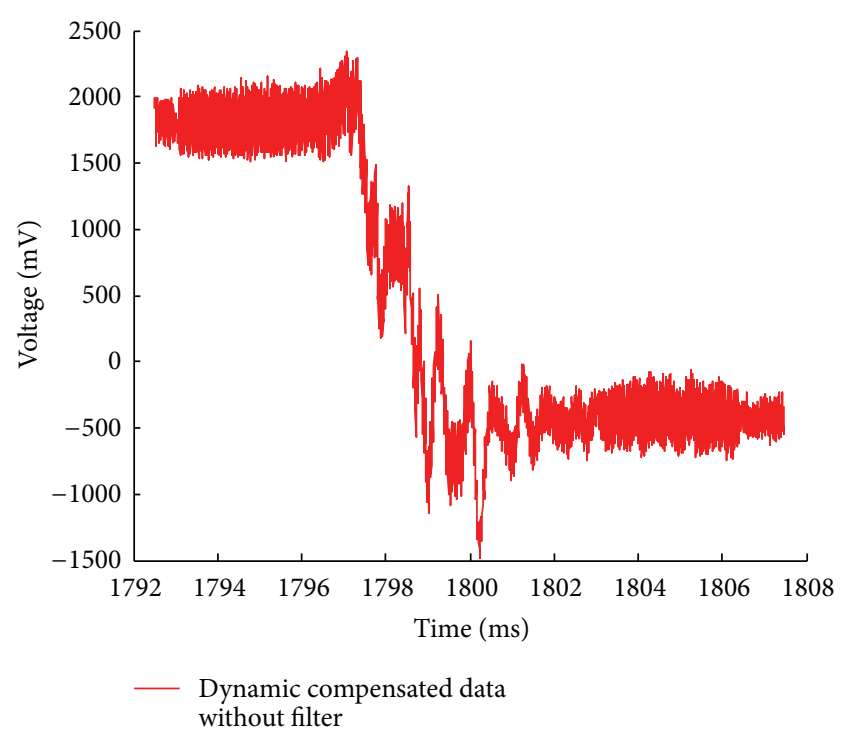

FIGURE 5: Dynamic compensation results of step response without low-pass filter.

The dynamic algorithm of the two-axis force sensor can be obtained as shown in (10):

$$
\begin{aligned}
y_{c}(n)= & \frac{1}{1+} 0.012 \\
& \times[3 \times(1+0.004) v(n)-3 \\
& \left.\quad \times v(n-1)+y_{c}(n-1)\right], \quad n=1,2,3 \ldots
\end{aligned}
$$

Figure 5 shows dynamic compensated data without the low-pass filter. The red line indicates the step response data after dynamic compensation but without a low-pass filter.

Comparing Figure 5 with Figure 4, the red line indicates that much more high-frequency noises are induced from the dynamic compensation algorithm. As a result, a low-pass filter is necessary after the dynamic compensation network.

The moving average filter is utilized as the low-pass filter. The length of the moving average filter kernel is defined as 100 , that is, $m=100$. The low-pass filter can be expressed as

$$
y_{L}(n)=\frac{1}{100} \sum_{i=n-50+1}^{n+50} y_{c}(n+i) \quad n=0,1,2, \ldots
$$

In order to make a contrast, both the original data as shown in Figure 4 and the dynamic compensated data as shown in Figure 5 are passed through the moving average filter. Figure 6 shows comparisons between original data and dynamic compensated data after the low-pass filter.

In Figure 6, the black line represents original data filtered by the low-pass filter. The red line represents dynamic compensated data filtered by the same low-pass filter. Figure 6 shows that the response time of the two-axis force sensor is reduced from about $4 \mathrm{~ms}$ to about $2.5 \mathrm{~ms}$. The time constant $\tau$ is reduced from about $1 \mathrm{~ms}$ to about $0.6 \mathrm{~ms}$.

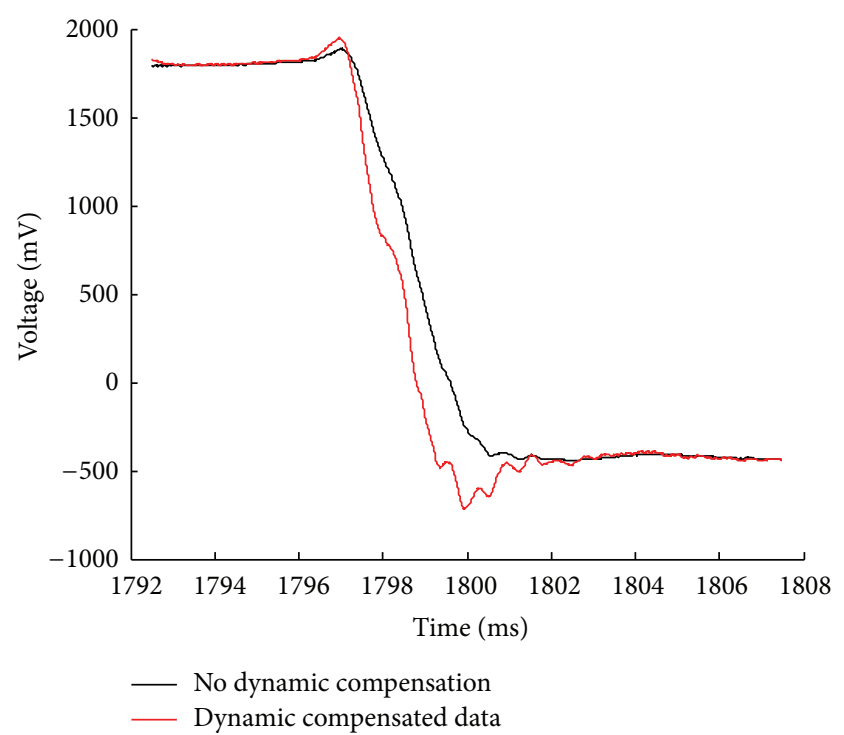

Figure 6: Comparisons between original data and dynamic compensated data after low-pass filter.

The unit step response function of the dynamic compensated two-axis force sensor can be expressed as

$$
f_{U}(t)=\left(1-e^{-(t-\lambda) /\left(6 \times 10^{-4}\right)}\right) U(t-\lambda),
$$

where $U$ represents the unit step function.

As a result, the dynamic characteristic of the two-axis force sensor is improved a lot from the proposed dynamic compensation method. The dynamic compensation method is simple and not subjected to local minimum.

\section{Conclusions}

In this paper, a dynamic compensation method to efficiently improve dynamic characteristic of two-axis force sensors is presented. The dynamic compensation method contains a dynamic compensation network and a low-pass filter. The two-axis force sensor is assumed as a first-order system. The dynamic compensation network, which is based on backward difference method, broadens the operation frequency of the force sensors. High-frequency noises may be intensified from the compensation network. A moving average filter is added to filter the high-frequency noises.

Dynamic calibration experiments are implemented. A negative step force signal is generated by sudden release of applying loads. The step response obtained from the calibration experiment demonstrates the rationality of the assumption of force sensors to be a first-order system. The proposed dynamic compensation method is applied in the experiment data. Experiment results demonstrate the effectiveness of our method. The adjusting time in the step response is reduced from about $4 \mathrm{~ms}$ to about $2.5 \mathrm{~ms}$. The time constant $\tau$ is reduced from about $1 \mathrm{~ms}$ to about $0.6 \mathrm{~ms}$.

Compared to the traditional dynamic compensation algorithm based on artificial neural network, our method is fast and not subjected to local minimum. The proposed dynamic 
compensation method can also be implemented in other multiaxis force sensors.

\section{Acknowledgment}

This work was supported by the National Natural Science Foundation of China (no. 61272379).

\section{References}

[1] J. Ma and A. Song, "Fast estimation of strains for cross-beams six-axis force/torque sensors by mechanical modeling," Sensors, vol. 13, pp. 6669-6686, 2013.

[2] A. Song, J. Wu, G. Qin, and W. Huang, "A novel self-decoupled four degree-of-freedom wrist force/torque sensor," Measurement, vol. 40, no. 9-10, pp. 883-891, 2007.

[3] Y.-J. Li, B.-Y. Sun, J. Zhang, M. Qian, and Z.-Y. Jia, "A novel parallel piezoelectric six-axis heavy force/torque sensor," Measurement, vol. 42, no. 5, pp. 730-736, 2009.

[4] M. S. Müller, L. Hoffmann, T. C. Buck, and A. W. Koch, "Fiber bragg grating-based force-torque sensor with six degrees of freedom," International Journal of Optomechatronics, vol. 3, no. 3, pp. 201-214, 2009.

[5] R. J. Wood, K.-J. Cho, and K. Hoffman, "A novel multi-axis force sensor for microrobotics applications," Smart Materials and Structures, vol. 18, no. 12, Article ID 125002, 2009.

[6] B. Siciliano and O. Khatib, Springer Handbook of Robotics, Springer, New York, NY, USA, 2008.

[7] T. Lefebvre, J. Xiao, H. Bruyninckx, and G. De Gersem, "Active compliant motion: a survey," Advanced Robotics, vol. 19, no. 5, pp. 479-499, 2005.

[8] G. Xu, A. Song, and H. Li, "Adaptive impedance control for upper-limb rehabilitation robot using evolutionary dynamic recurrent fuzzy neural network," Journal of Intelligent and Robotic Systems, vol. 62, no. 3-4, pp. 501-525, 2011.

[9] F. Nagata, Y. Kusumoto, K. Watanabe et al., "Polishing robot for PET bottle molds using a learning-based hybrid position/force controller," in Proceedings of the 5th Asian Control Conference, pp. 914-921, July 2004.

[10] K.-J. Xu and C. Li, "Dynamic decoupling and compensating methods of multi-axis force sensors," IEEE Transactions on Instrumentation and Measurement, vol. 49, no. 5, pp. 935-941, 2000.

[11] Y. Altintas and S. S. Park, "Dynamic compensation of spindleintegrated force sensors," CIRP Annals-Manufacturing Technology, vol. 53, no. 1, pp. 305-308, 2004.

[12] K.-J. Xu, C. Li, and Z.-N. Zhu, "Dynamic modeling and compensation of robot six-axis wrist force/torque sensor," IEEE Transactions on Instrumentation and Measurement, vol. 56, no. 5, pp. 2094-2100, 2007.

[13] A. Yu, W. Huang, and G. Qin, "Dynamic modeling and compensation method based on genetic neural network for new type robot wrist force sensor," Chinese Journal of Mechanical Engineering, vol. 42, no. 12, pp. 239-244, 2006.

[14] S. Chen and S. A. Billings, "Neural networks for nonlinear dynamic system modelling and identification," International Journal of Control, vol. 56, pp. 319-346, 1992.

[15] P. J. Brockwell and R. A. Davis, Time Series: Theory and Methods, Springer, New York, NY, USA, 2009.
[16] J. Ma and A. Song, "Development of a novel two-axis force sensor for chinese massage robot," Applied Mechanics and Materials, vol. 103, pp. 299-304, 2012.

[17] N. Hu, H. Fukunaga, S. Matsumoto, B. Yan, and X. H. Peng, "An efficient approach for identifying impact force using embedded piezoelectric sensors," International Journal of Impact Engineering, vol. 34, no. 7, pp. 1258-1271, 2007.

[18] M. Tracy and F.-K. Chang, "Identifying impact load in composite plates based on distributed piezoelectric sensor measurements," in Smart Structures and Materials: Smart Structures and Integrated Systems, vol. 2717 of Proceedings of SPIE, pp. 231-236, February 1996.

[19] M. P. Schoen, "Dynamic compensation of intelligent sensors," IEEE Transactions on Instrumentation and Measurement, vol. 56, no. 5, pp. 1992-2001, 2007. 



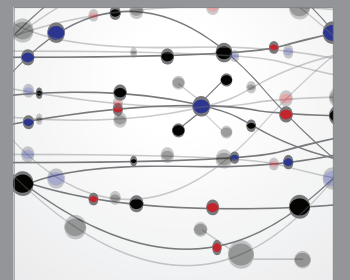

The Scientific World Journal
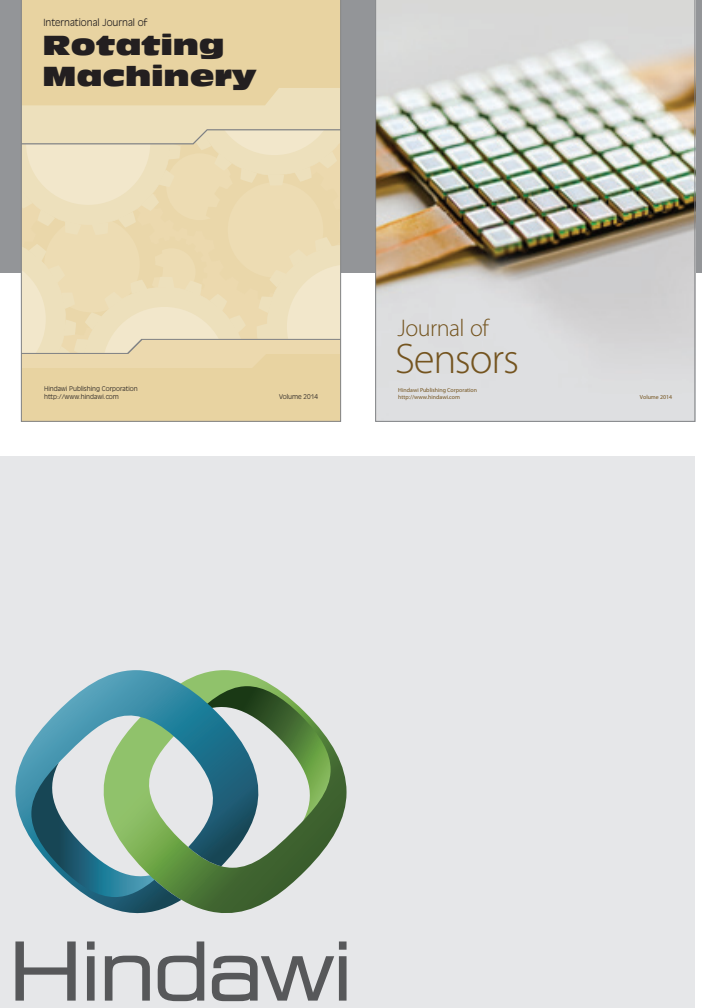

Submit your manuscripts at http://www.hindawi.com

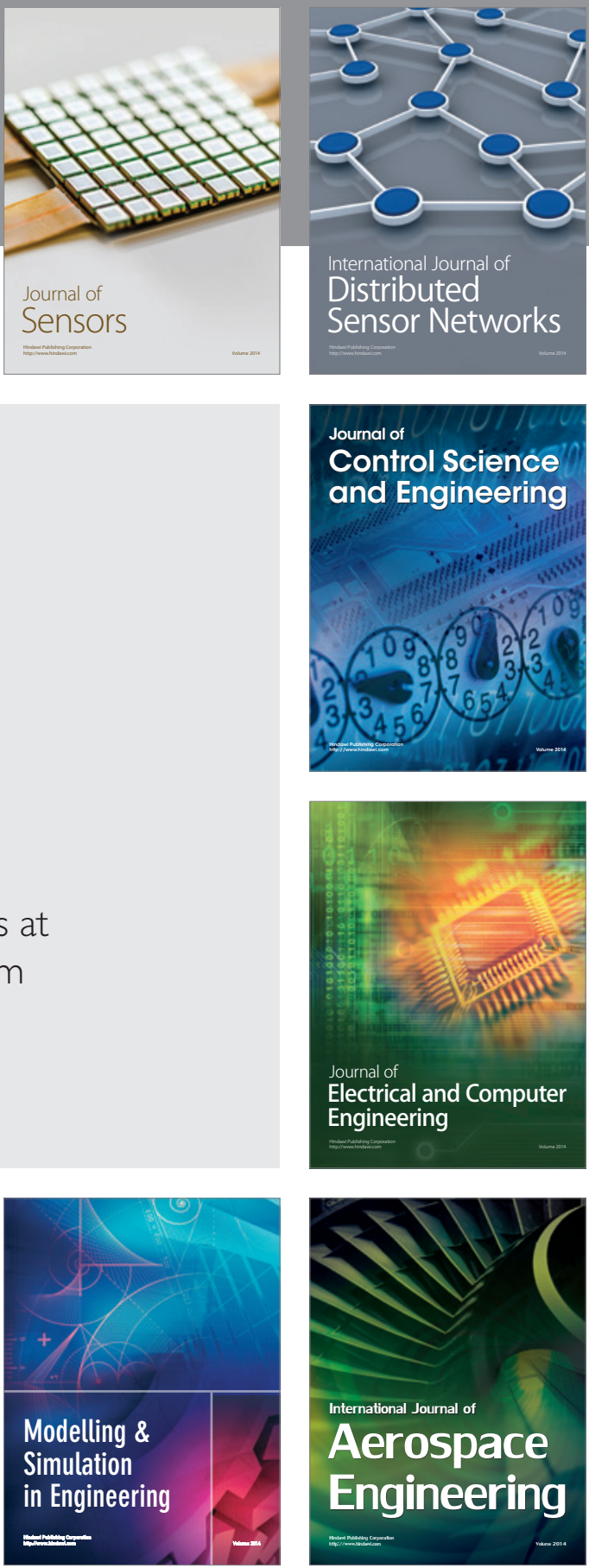

Journal of

Control Science

and Engineering
